\title{
Desarrollo de curvas de deterioro para pavimento flexible y factor de incertidumbre
}

\section{Development of deterioration curves for flexible pavement and uncertainty factor}

\section{Greivin Picado Muñoz}

Universidad de Costa Rica, San José-Costa Rica,

g.picadomunoz@gmail.com

Fecha de recepción: 14 de marzo de 2016 / Fecha de aprobación: 12 de abril de 2016

\section{RESUMEN}

Debido al problema de planificación que existe a nivel de Red Vial Cantonal en Costa Rica, gran cantidad de caminos cuentan con un nivel avanzado de deterioro y reciben actividades de mantenimiento que no son adecuadas, lo cual se traduce en un alto costo para los gobiernos locales. Esto se debe a que muchas de las Unidades Técnicas de Gestión Vial Municipal no cuentan con estudios e información básica necesaria para generar las curvas de deterioro que permitan establecer un modelo e identificar el estado de las carreteras.

Con el presente trabajo se busca realizar una evaluación de los caminos de la Gran Área Metropolitana, en las provincias de San José, Alajuela, Cartago y Heredia; de manera que se pueda conocer la condición en la que se encuentran, así como, obtener las curvas de deterioro a partir de la información recolectada en campo.

Para la auscultación visual de los deterioros y el cálculo del Î́ndice de Condición de Pavimento, fue empleada la metodología de la norma ASTM D-6433 y posteriormente, utilizando un análisis estadístico multivariable de los datos obtenidos, se generan las curvas de deterioro para distintas condiciones de los caminos de la Red Vial Cantonal.

Con las curvas de deterioro encontradas y la ecuación que incluye el factor de incertidumbre se obtiene una herramienta muy útil para generar un modelo de deterioro de los caminos, de manera que los encargados de la planificación en las Unidades Técnicas, puedan definir el tipo y tiempo óptimo de las intervenciones requeridas, así como una adecuada administración de los recursos.

PALABRAS CLAVES: Deterioros en pavimento flexible, Índice de Condición de Pavimento, curvas de deterioro, actividades de mantenimiento vial.

\section{ABSTRACT}

Due to planning issues in the Local Road Network of Costa Rica, an important amount of roads have an advanced level of distress and receive inappropriate maintenance activities, which implies a high cost for local governments. This is because several of the Road Management Units do not have the studies and basic information needed to generate the deterioration curves that would allow to identify the state of the roads.

The following project seeks to make an assessment of the roads in the Metropolitan Area, in the provinces of San José, Alajuela, Cartago and Heredia; in order to recognize the roads' condition and develop the deterioration curves from the information collected in field.

The ASTM D-6433 Standard was applied for the field condition survey of the distress and the calculating of the Pavement Condition Index. Next, the deterioration curves where created by using a multivariate statistical analysis of the data obtained.

Having found the deterioration curves and the equation that includes the uncertainty factor, an useful tool is obtained, which can generate a deterioration model of the roads, allowing the department in charge to establish the optimal timing of pavement preventive maintenance, the adequate maintenance activities according to the level of distress and a proper distribution of the public resources.

KEY WORDS: Flexible pavement distresses, Pavement Condition Index, deterioration curves, maintenance activities. 


\section{INTRODUCCIÓN}

El desarrollo de infraestructura vial en Costa Rica, a nivel cantonal, sufre un problema de planificación que inició hace varias décadas, cuando fueron construidos gran parte de los caminos que se utilizan actualmente. Muchos de ellos surgieron a partir de pequeños proyectos cuyo principal objetivo era el de resolver las necesidades inmediatas de una creciente población, sin tener en cuenta un plan que permitiera su gestión a mediano o largo plazo.

En años anteriores, los niveles de tráfico (TPD) y el porcentaje de vehículos pesados era relativamente bajo, por lo que pocas veces se realizó un diseño formal en los proyectos; y no se dio la importancia adecuada a la implementación de controles de calidad al momento de la construcción. Esto genera que las cargas que existen en la actualidad, comprometan las estructuras de pavimentos y en ocasiones generen un deterioro acelerado de los caminos.

López (2010) llegó a la conclusión de que la condición estructural de las vías cantonales es deficiente (mala o severamente deteriorado), tal y como se puede observar en la Figura 1, existe un marcado problema con la condición de los pavimentos, donde más del $60 \%$ de la red se encuentra en una condición de deterioro severo, lo que da como conclusión que se requiere de una intervención significativa. Ingenieros expertos en el tema definen como una prioridad el mejoramiento del IRI y del índice de condición de pavimento $(\mathrm{PCI})$.

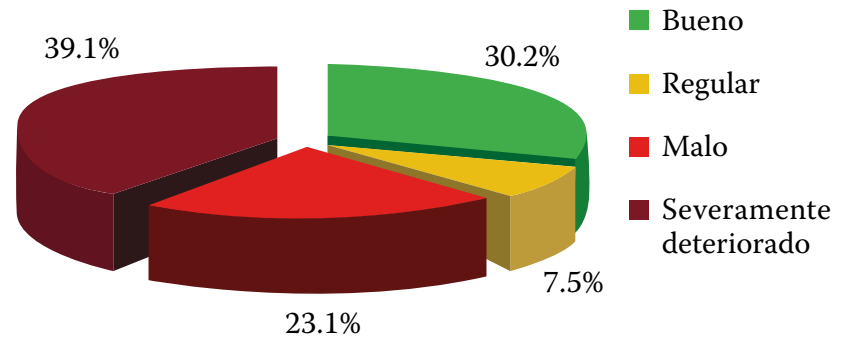

Figura 1. Condición estructural en la red cantonal evaluada Fuente: López, 2010

En Costa Rica se cuenta con poca información acerca del estado de la Red Vial Cantonal, que constituye la mayor parte de las carreteras del país (cerca de un $80 \%$ ); y no existen estudios con parámetros internacionalmente comparables como el Índice de Rugosidad Internacional (IRI), estudios de deflectometría (FWD), Índice de Condición de Pavimento (PCI) u otros.

Es entonces donde se dificulta la labor de planificación, puesto que no se conoce cuál es la condición de cada camino, ni como se deteriora a lo largo del tiempo, dificultando la priorización de las intervenciones de conservación vial, rehabilitación, reconstrucción; o bien, definir la necesidad de nuevos proyectos.

El conocimiento del tiempo adecuado para llevar a cabo una intervención es un factor muy importante, ya que muchas veces se procede con estos trabajos, una vez que el estado de las carreteras es deplorable. Incluso, por desconocimiento del comportamiento de las vías a lo largo del tiempo, se pueden aplicar técnicas de intervención que no son las más adecuadas, según el estado en que se encuentra el camino. Todo esto implica un gran costo económico, puesto que se deben realizar intervenciones mayores, con un costo superior, en comparación con intervenciones adecuadas, en el momento oportuno y a un costo menor.

La importancia del presente estudio radica en que la información generada es necesaria para comprender de una mejor manera cómo se encuentran las carreteras actualmente y su comportamiento a lo largo del tiempo. El método utilizado permite acercarse al problema y establecer una caracterización visual de los tipos de deterioros existentes. Con la información obtenida en campo y los historiales de intervención de las carreteras, se pueden generar las denominadas curvas de deterioro.

Como se puede observar en la Figura 2, las curvas permiten tener una mejor conceptualización de las condiciones que se están presentando en un momento dado; y a la vez predecir la condición a futuro. Esto implica un aporte muy significativo en lo que al tema de administración de la infraestructura vial se refiere, ya que se podrá definir la intervención que se requiere para cada tipo de carretera.

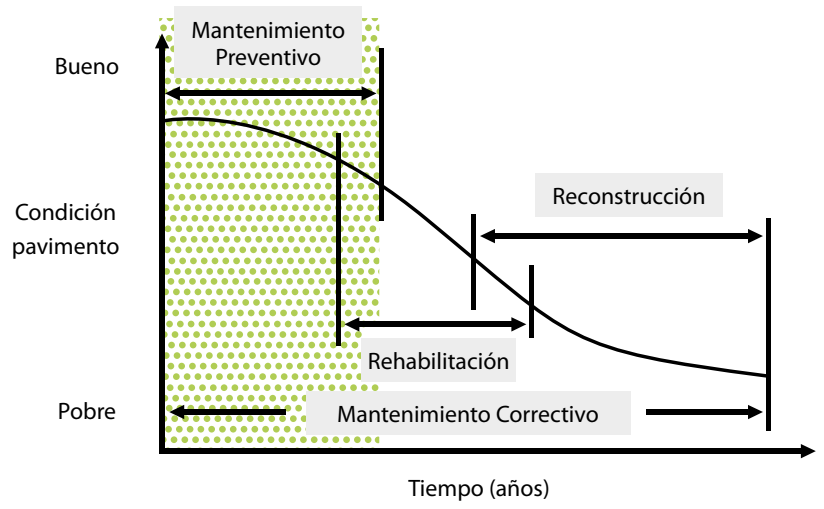

Figura 2. Relación entre condición de pavimento y tipos de tratamiento (IDOT, 2012) 
Es por ello que el objetivo general del proyecto consiste en desarrollar las curvas de deterioro para la Red Vial Cantonal en pavimento flexible, a partir de la información recolectada en campo para las carreteras en estudio. Utilizando como base la norma ASTM D-6433 para las mediciones de los deterioros y el cálculo del Índice de Condición de Pavimento (PCI).

\section{OBJETIVOS}

- Realizar la inspección visual de caminos de la Red Vial Cantonal, de acuerdo con la metodología ASTM D-6433 y estimar los Índices de Condición de Pavimento (PCI).

- Emplear la metodología del análisis de Clúster para agrupar los datos según el tipo de estructura presente en las carreteras.

- Generar las curvas de deterioro para pavimentos flexibles, utilizando los Índices de Condición de Pavimento y el año de última intervención en conservación vial.

- Determinar el factor de incertidumbre para la ecuación del modelo de deterioro obtenido.

\section{METODOLOGÍA}

\section{Fase Preliminar}

Esta etapa se realizó previa a la visita de campo, dando inicio con la recolección de la información básica para el estudio. A cada una de las Unidades Técnicas de Gestión Vial Municipal se solicitaron datos como el nombre del camino, ubicación, TPD, tipo de estructura del pavimento, la fecha de construcción y los tipos de intervenciones en conservación vial que se hayan realizado en los últimos años. Con la información recolectada se generó una base de datos de las carreteras.

\section{Fase Desarrollo}

Posteriormente se procedió con el trabajo de campo. En el desarrollo del proyecto se empleó como base la metodología de la norma ASTM D-6433 (ASTM, 2014), para la auscultación de los deterioros en campo, el cálculo de las Unidades de Muestreo (UM) y su respectivo Índice de Condición de Pavimento.

Cuando se visitaron los proyectos, se midió el ancho promedio de la calzada para determinar las dimensiones de las Unidades de Muestreo, la cantidad total de UM existente en la sección de carretera que se está evaluando y el respectivo intervalo de muestreo. Con estos datos se procede con la inspección de los deterioros existentes en la carretera (FHWA, 2003), clasificándolos por tipo, cantidad y severidad.

Una vez que se genera esta información, se procede con el cálculo del PCI para cada una de las unidades de muestreo y de manera complementaria se estima un promedio ponderado para la carretera, tomando en cuenta la condición general del camino.

Con este parámetro y la fecha de la última intervención en conservación vial realizada en la carretera, se obtiene una dispersión de los datos de manera que, aplicando un análisis estadístico de Clúster, se pueden desarrollar las curvas de deterioro para diferentes condiciones.

Para el análisis de Clúster, fue utilizado el programa de análisis estadístico multivariable PAST, el cual permite generar grupos de puntos que se comportan de manera similar, considerando la variable de PCI para cada camino y el año de última intervención. En cada grupo obtenido se aplicó una curva de mejor ajuste de manera que se pudieron desarrollar las curvas de deterioro para caminos con estructura suficiente, media e insuficiente.

Finalmente, se realizó un análisis estadístico de la regresión obtenida para comprobar el modelo seleccionado como ideal y posteriormente se empleó una metodología de análisis de residuos con el fin de definir el factor de incertidumbre.

\section{Fase Final}

Esta etapa comprendió el análisis de la información obtenida, de manera que se definieron las conclusiones del trabajo. En resumen, la metodología empleada en el trabajo se describe en la Figura 3. 


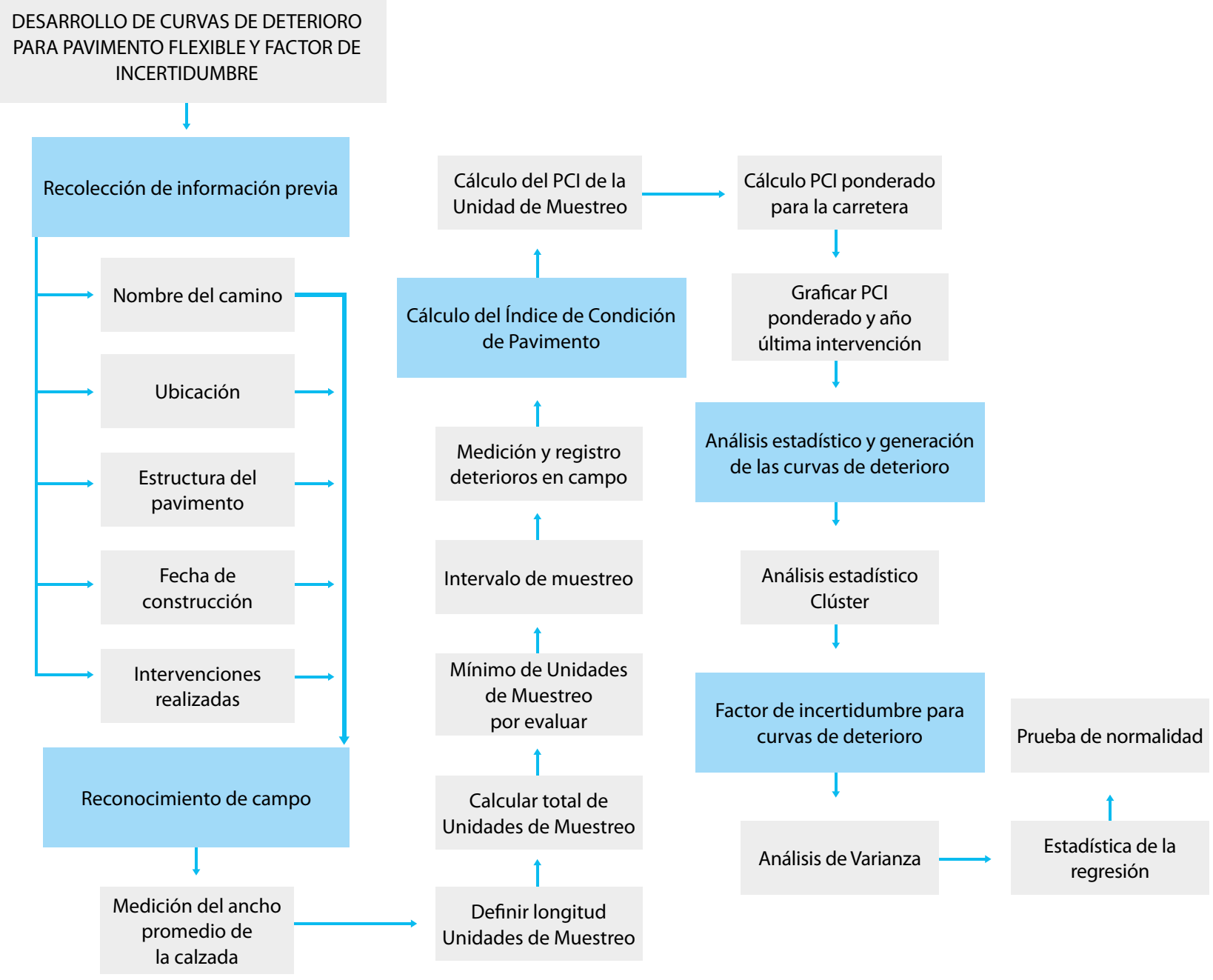

Figura 3. Metodología de trabajo para desarrollo curvas de deterioro en pavimento flexible

\section{RESULTADOS}

Para la toma de decisiones en el proceso de planificación, donde los gobiernos locales requieren conocer el comportamiento mediante curvas de deterioro de los pavimentos flexibles en los caminos cantonales, se encontró una ecuación correspondiente a la curva típica de deterioro para pavimentos con estructura suficiente, a la cual se le puede asignar un factor de incertidumbre, dependiendo de la confianza que se desea de los datos por parte del planificador.

\section{Evaluación De Los Caminos}

En total se llevó a cabo la revisión de 188 caminos, en las provincias de San José, Alajuela, Cartago y Heredia, como se muestra en la Tabla 1. De la Red Vial Cantonal que forma parte del Gran Área Metropolitana (GAM), existen cerca de 3418 km asfaltados, según datos de la Dirección de Planificación Sectorial del Ministerio de Obras Públicas y Transportes, de manera que los proyectos seleccionados para llevar a cabo su evaluación completaron un total de $100 \mathrm{~km}$, lo cual representa un 3\% de todos los caminos asfaltados del GAM. 
Tabla 1. Desglose de los caminos evaluados

\begin{tabular}{|c|c|c|c|c|c|c|}
\hline Provincia & $\begin{array}{c}\text { RVC Longitud } \\
\text { en km (1) }\end{array}$ & $\mathrm{km}$ Asfaltados (2) & $\begin{array}{c}\text { \% Asfaltados } \\
\text { RVC-GAM (1)/(2) }\end{array}$ & $\begin{array}{c}\text { Cantidad } \\
\text { de caminos } \\
\text { Evaluados }\end{array}$ & km Evaluados (3) & $\begin{array}{c}\text { \% km Asfaltados } \\
\text { Evaluados (2)/(3) }\end{array}$ \\
\hline San José & 2725.37 & 1416.09 & $51.96 \%$ & 62 & 25.04 & $1.77 \%$ \\
\hline Alajuela & 1940.11 & 682.43 & $35.17 \%$ & 23 & 16.26 & $2.38 \%$ \\
\hline Cartago & 1559.70 & 597.15 & $38.29 \%$ & 47 & 27.19 & $4.55 \%$ \\
\hline Heredia & 936.73 & 723.01 & $77.18 \%$ & 56 & 31.52 & $4.36 \%$ \\
\hline
\end{tabular}

\section{Desarrollo De Las Curvas De Deterioro}

Para cada uno de los caminos descritos anteriormente se llevó a cabo el cálculo del Índice de Condición de Pavimentos de acuerdo a la metodología ASTM D-6433. Al comparar estos resultados con el año respectivo de la última intervención en conservación vial se obtiene una gráfica como se presenta en la Figura 4.

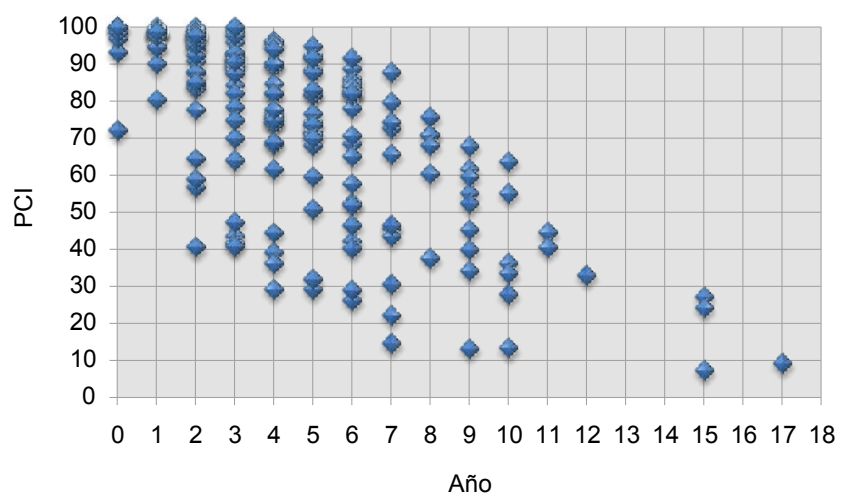

Figura 4. Gráfica PCl vs año para todos los caminos

Tras un análisis detallado de los datos de la Figura 4, se pudieron indentificar tres tendencias de deterioro a lo largo del tiempo. Cada una de ellas, se puede relacionar con la estructura presente en el camino. En la parte inferior del conjunto de datos, se puede observar el caso de carreteras con una estructura deficiente, donde es esperable encontrar datos de PCI bajos en periodos cortos de tiempo. Así como, en la parte superior de la dispersión, caminos que cuentan con una estructura suficiente y un diseño adecuado.

Es entonces cuando se buscó la división de los datos, basándose en un análisis estadístico. En este caso, se aplicó un análisis multivariable de "Clúster" (Balzarini et al., 2014), cuyo principal objetivo es agrupar los datos según las distancias que existen entre ellos y establecer jerarquías entre los conjuntos. Es decir, separar de manera gráfica, los grupos de datos que son estadísticamente similares.

Para el trabajo se empleó un análisis de tipo jerárquico para establecer los grupos de datos y la metodología de encadenamiento promedio (UPGMA) para el cálculo de las distancias entre los pares de datos, ya que a partir de este procedimiento se obtuvo un mejor coeficiente de correlación cofenético.

La metodología se aplicó para cada uno de los años en estudio, de manera que luego de agrupar los resultados obtenidos en el análisis, se separó la totalidad de los proyectos en tres conjuntos diferentes denominados: caminos con estructura suficiente, estructura media y estructura insuficiente. Para cada uno de ellos se llevó a cabo un análisis estadístico, de manera que se puede observar el comportamiento existente en los grupos generados. La Tabla 2, muestra las características generales de los conjuntos.

Tabla 2. Propiedades estadísticas de los grupos de datos por tipo de estructura

\begin{tabular}{|c|c|c|c|c|}
\cline { 2 - 5 } \multicolumn{1}{c|}{} & $\begin{array}{c}\text { Cantidad } \\
\text { Observaciones }\end{array}$ & $R^{2}$ & Promedio & $\begin{array}{c}\text { Desviación } \\
\text { Estándar }\end{array}$ \\
\hline $\begin{array}{c}\text { Estructura } \\
\text { Suficiente }\end{array}$ & 129 & 0,878 & 84,00 & 6,439 \\
\hline $\begin{array}{c}\text { Estructura } \\
\text { Media }\end{array}$ & 39 & 0,748 & 60,00 & 9,755 \\
\hline $\begin{array}{c}\text { Estructura } \\
\text { Insuficiente }\end{array}$ & 20 & 0,847 & 38,00 & 6,939 \\
\hline
\end{tabular}

A partir de los resultados obtenidos en la Tabla 2, se puede garantizar que la división de los proyectos en 3 grupos de carreteras permite ajustar adecuadamente los datos, de manera que se pueden generar las curvas de deterioro que se muestran a continuación, aplicando una curva de mejor ajuste en cada caso.

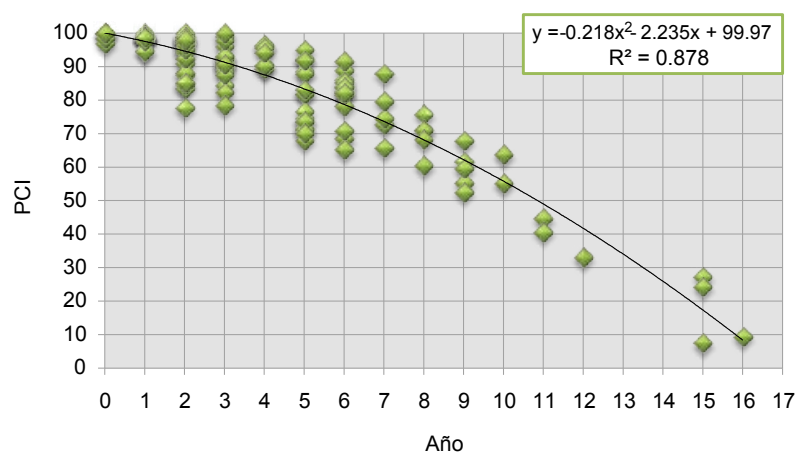

Figura 5. Curva de Deterioro, caminos estructura suficiente 
Como se puede observar en la Figura 5, los caminos que cuentan con una estructura suficiente mantienen una mejor condición por periodos de tiempo mayores. Según el Manual de Mantenimiento y Rehabilitación de Pavimentos Flexibles (Jugo 2005), existe una zona denominada "óptima de rehabilitación". En este periodo de tiempo, aunque en la carretera se pueden encontrar algunos deterioros, el pavimento tiene gran parte de su capacidad para soportar las cargas, por lo que las intervenciones realizadas ayudarán a mejorar considerablemente su condición.

La fase comprendida entre el momento en que se pone en operación un pavimento y esta zona (PCI 55 \pm 7), se da una reducción aproximada de un $40 \%$ de su calidad, consumiendo casi un $75 \%$ de la vida del pavimento en términos de repeticiones de cargas. Si se analiza la curva de deterioro obtenida para los caminos con una estructura suficiente, se puede observar que esta zona se aproxima a los 10 años, si aplican las actividades de mantenimiento adecuadamente.

Por otro lado, los caminos con una estructura media (Figura 6) presentan una reducción en la condición de las carreteras durante los primeros años y por tanto la curva se desplaza de manera que las actividades de mantenimiento rutinario deben iniciar en un periodo de tiempo menor, cercano a los 3 años. Además, debido al comportamiento encontrado en este tipo de caminos, la necesidad de una rehabilitación se establece cuando se cumplen cerca de 6 años del momento en que se pone en operación.

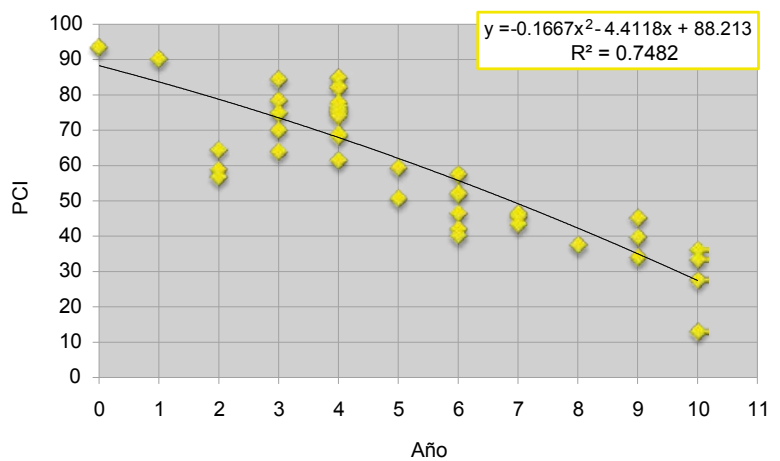

Figura 6. Curva de Deterioro, caminos estructura media

Si se analiza la Figura 7, correspondiente a los caminos con una estructura insuficiente, se puede observar un decrecimiento acelerado de la condición del pavimento a lo largo del tiempo y por tanto, en solo 2 años se puede observar una pérdida de hasta un $40 \%$. Incluso, este tipo de caminos comienzan a presentar síntomas importantes de deterioro a los pocos meses, requiriendo actividades de mantenimiento rutinario en un periodo de tiempo casi inmediato, comparado con el momento en que se pone en operación.
El comportamiento encontrado en la Figura 7, es producto de carreteras que cuentan con un alto nivel de deterioro, las cuales, en lugar de recibir algún mejoramiento en su estructura, se les interviene con soluciones que no son las más adecuadas a su condición, de manera que su estado vuelve a caer rápidamente.

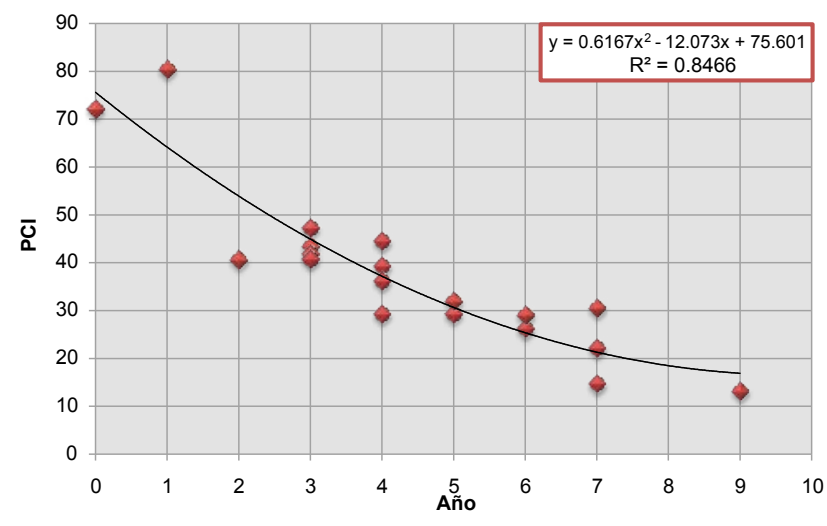

Figura 7. Curva de Deterioro, caminos estructura insuficiente

\section{Factor De Incertidumbre Para Curvas De Deterioro}

En el caso de que exista desconocimiento del comportamiento de los caminos cantonales, se puede emplear una corrección de la curva de deterioro basándose en un indicador de variabilidad, de manera que se suma un parámetro de la forma $\mathrm{Z}_{0} \sigma$ a la regresión. Debido al comportamiento encontrado en carreteras con una estructura suficiente, se tomó su ecuación como la ideal para generar el modelo de deterioro. Para comprobar la regresión obtenida se realizó un análisis de varianza, con la hipótesis nula $\left(\mathrm{H}_{0}\right)$ : el modelo no explica el fenómeno en estudio.

Tabla 3. Análisis de varianza

\begin{tabular}{|c|c|c|}
\cline { 2 - 3 } \multicolumn{1}{c|}{} & Coeficientes & Probabilidad \\
\hline Intercepto & 999.710 & $2,00 \mathrm{E}-106$ \\
\hline Variable X & -22.359 & $1,19 \mathrm{E}-02$ \\
\hline Variable X & -0.2181 & $7,91 \mathrm{E}-05$ \\
\hline \multirow{2}{*}{ Regresión } & $\mathrm{F}$ & Valor Crítico F \\
\cline { 2 - 3 } & 455.32 & $2,18 \mathrm{E}-55$ \\
\hline
\end{tabular}

Como se puede observar en la Tabla 3; a partir de un análisis de varianza con un nivel de significancia del 95\%, se encontró que la probabilidad de cada una de las variables $\mathrm{X}, \mathrm{X}^{2}$ e intercepto es pequeño, por lo tanto cada una de ellas es un parámetro significativo. Si se evalúa la totalidad de la regresión, se puede verificar como el valor de $\mathrm{F}$ es mucho mayor que el valor crítico de $\mathrm{F}$ y por tanto se rechaza la $\mathrm{H}_{0}$. De esta manera se acepta el modelo obtenido para carreteras con una estructura suficiente.

Posteriormente, se realizó un análisis estadístico de la regresión obtenida (Tabla 4), evaluando los residuos asociados, por medio 
de la herramienta de análisis de datos del programa Microsoft Excel. De esta manera, el error típico encontrado corresponde al valor de $\sigma$.

\section{Tabla 4. Estadística de la regresión}

\begin{tabular}{|c|c|}
\hline Coeficiente de determinación $\mathbf{R} \wedge \mathbf{2}$ & 0.8785 \\
\hline $\mathbf{R} \wedge \mathbf{2}$ ajustado & 0.8765 \\
\hline Error típico & 6.4386 \\
\hline Observaciones & 129 \\
\hline
\end{tabular}

De forma complementaria, se llevó a cabo una verificación del comportamiento de los residuos encontrados, para conocer si los datos responden a una distribución normal. En este caso se plantea una $\mathrm{H}_{0}$ : los residuos provienen de una población normalmente distribuida.

La prueba de normalidad se llevó a cabo por medio del programa de análisis estadístico PAST, a partir del cual se obtuvieron los resultados que se muestran en la Tabla 5.

\section{Tabla 5. Prueba de normalidad}

\begin{tabular}{|c|c|}
\cline { 2 - 2 } \multicolumn{1}{c|}{} & Resultado \\
\hline N & 129 \\
\hline Shapiro-Wilk W & 0.966 \\
\hline p(normal) & 0.002529 \\
\hline
\end{tabular}

Dado que el estadístico W de la prueba Shapiro-Wilk no es pequeño, se acepta la hipótesis nula y por tanto se puede asumir que el comportamiento de los residuos corresponde a una distribución normal. De manera complementaria, se realizó la prueba gráfica Normal Q-Q (Figura 8), donde al analizar el comportamiento de los residuos se comprueba su normalidad y por tanto el valor de Z0 se define a partir de los valores de la tabla normal estándar según el nivel de incertidumbre seleccionado (Tabla 6).

\section{Q-Q Normal}

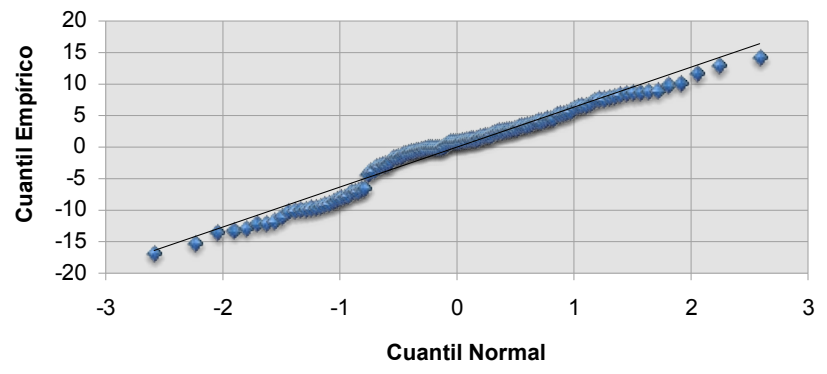

Tabla 6. $Z_{\circ}$ para diferentes niveles de Incertidumbre

\begin{tabular}{|c|c|}
\hline Incertidumbre & $\mathbf{Z}_{0}$ \\
\hline $99 \%$ & -2.57583 \\
\hline $95 \%$ & -1.95996 \\
\hline $90 \%$ & -1.64485 \\
\hline $85 \%$ & -1.43953 \\
\hline $80 \%$ & -1.28155 \\
\hline $75 \%$ & -1.15035 \\
\hline
\end{tabular}

De esta manera se puede emplear la curva de deterioro para caminos en pavimento flexible, de la forma:

$$
y=-0.2181 X^{2}-2.2359 X+100+6.4386 Z_{0}
$$

El nivel de incertidumbre aplicado en la Ecuación 1 puede variar dependiendo de la importancia del camino. Esta puede estar sujeta a diferentes parámetros como su ubicación y el nivel de tráfico; de manera que para las carreteras con un mayor nivel de importancia, se puede emplear un modelo de deterioro con un factor de incertidumbre mayor.

Otra forma de definir el parámetro $Z_{0}$ de la Ecuación 1, está relacionada con el conocimiento de la red en estudio por parte del planificador. En caso de que exista un total desconocimiento de las condiciones que se encuentran presentes, se puede emplear inicialmente un factor mayor e ir ajustando el mismo conforme se va dando seguimiento a los caminos a lo largo del tiempo.

La idea principal de los modelos para cada nivel de incertidumbre es tener una herramienta que permita establecer la forma en que se van deteriorando los caminos a lo largo del tiempo y a partir de ella, definir los tiempos óptimos de intervención de acuerdo a la condición en la que se encuentran. Posteriormente, luego de un proceso de evaluación de la red se puede ir ajustando el nivel de incertidumbre seleccionado, hasta alcanzar la curva de deterioro óptima encontrada para caminos con estructura suficiente.

En la Figura 9, se pueden observar las curvas de deterioro para cada nivel de incertidumbre; así como los tipos de actividades de mantenimiento vial. Cabe mencionar que una vez que se pone en operación una carretera se debe de iniciar con las actividades de mantenimiento preventivo de manera que se evite un decrecimiento acelerado de la calidad de los caminos.

Figura 8. Prueba gráfica Normal Q-0 


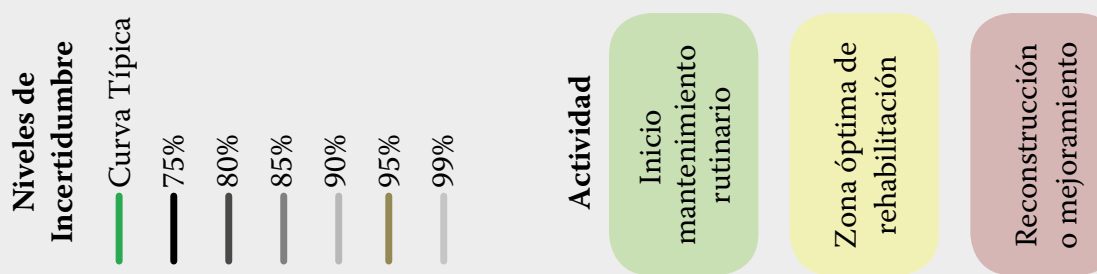

今

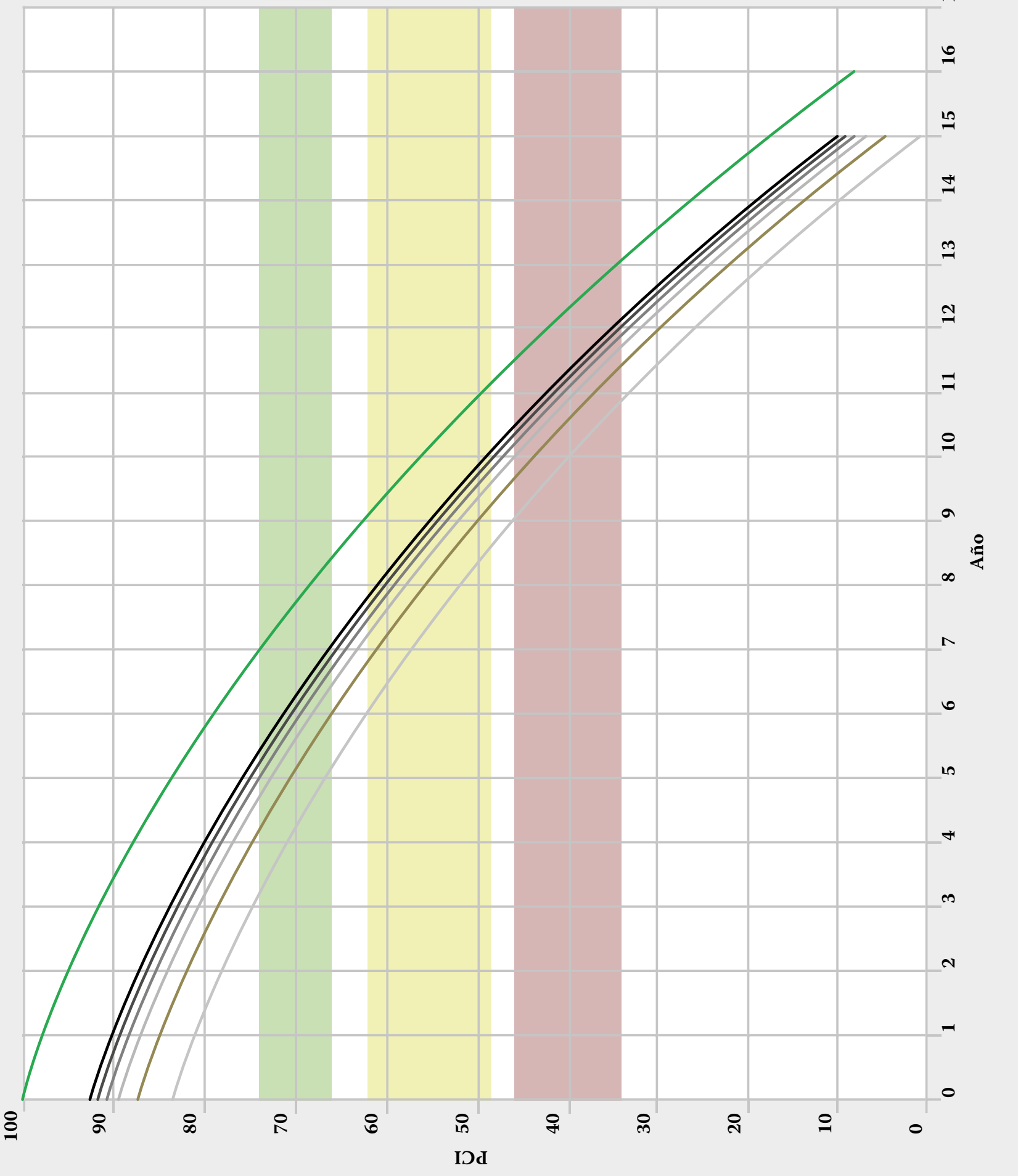

Figura 9. Curvas de Deterioro por Nivel de Incertidumbre 


\section{CONCLUSIONES}

1. En varias de las Unidades Técnicas de Gestión Vial Municipal, se encontró un problema con el manejo de la información, puesto que no se lleva un registro detallado de las intervenciones que se realizan, incluso no se tienen datos de la estructura, el año de construcción. Esta situación limita la posibilidad que las UTGVM, puedan desarrollar sus propias curvas de deterioro con parámetros comparables, para una mejor administración de la red de caminos a su cargo.

2. El desconocimiento del estado actual y la condición previa de las carreteras (historial de intervenciones), genera que se destinen los recursos de manera inapropiada. En ocasiones, se realizan intervenciones que no son las más adecuadas al tipo de deterioro que presentan las carreteras, lo que se traduce en una pérdida importante en el nivel de servicio a lo largo de un periodo corto de tiempo y en un costo económico significativo.

3. En el caso que la UTGVM no cuente con las respectivas curvas de deterioro con parámetros comparables, tendrá la posibilidad de emplear la Ecuación (1), a la que se llegó en este estudio y que permitirá obtener las curvas de deterioro de acuerdo con el grado de incertidumbre que se estime apropiado.

4. Se pudo observar la importancia de las curvas de deterioro para la administración de las estructuras de pavimento, puesto que permiten conocer el tiempo oportuno de intervención, reduciendo los costos y optimizando los recursos.

5. Existe una cantidad importante de caminos que cuentan con una estructura insuficiente, lo que genera que haya un deterioro del camino de manera acelerada, generando una pérdida de aproximadamente un $40 \%$ de su calidad a los dos años de su puesta en operación.

6. A las carreteras que cuentan con estructura insuficiente, se les interviene con soluciones que no son las más adecuadas, de manera que su estado vuelve a caer rápidamente. Si se visualiza este tipo de caminos a largo plazo, su costo de mantenimiento es mayor en comparación a si se hubiera realizado un mejoramiento oportuno en la vía.

\section{REFERENCIAS}

1. American Standard Testing Materials (ASTM). (2014). Standar Practice for Roads and Parking Lots Pavement Condition Index. Washington, D.C.

2. Balzarini, M., Bruno, C., \& Córdoba, M. (2014). Análisis de Conglomerado. Córdoba, Argentina: Universidad Nacional de Córdoba.

3. Federal Highway Administration. (2003). Distress Identification Manual. Washington D.C.: US. Department of Transportation.

4. Illinois Department of Transportation (IDOT). (2012). Bureau of Local Roads \& Streets Manual. Springfield, Illinois.

5. López, S. (2010). Diagnóstico de la red vial cantonal pavimentada. Congreso CIC-2010. San José, Costa Rica. Obtenido de Infraestructura Vial Digital: http://www.lanamme.ucr. ac.cr/riv/index.php?option=com_content\&view=article\&id=279\&Item id $=324$

6. Jugo, A. (2005). Manual de Mantenimiento y Rehabilitación de Pavimentos Flexibles. Caracas. 